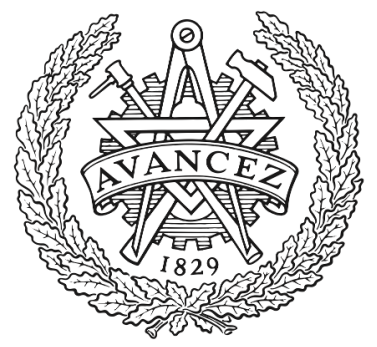

CHALMERS

UNIVERSITY OF TECHNOLOGY

\title{
Per-Antenna Power Distribution of a Zero-Forcing Beamformed ULA in pure LOS MU-MIMO
}

Downloaded from: https://research.chalmers.se, 2023-04-26 10:03 UTC

Citation for the original published paper (version of record):

Amani, N., Glazunov, A., Ivashina, M. et al (2018). Per-Antenna Power Distribution of a

Zero-Forcing Beamformed ULA in pure LOS MU-MIMO. IEEE Communications Letters, 22(12):

2515-2518. http://dx.doi.org/10.1109/LCOMM.2018.2871828

N.B. When citing this work, cite the original published paper.

C2018 IEEE. Personal use of this material is permitted.

However, permission to reprint/republish this material for advertising or promotional purposes 


\title{
Per-Antenna Power Distribution of a Zero-Forcing Beamformed ULA in pure LOS MU-MIMO
}

\author{
Navid Amani, Andrés Alayón Glazunov, Marianna V. Ivashina, and Rob Maaskant
}

\begin{abstract}
An analytical model is presented to determine the perantenna power distribution of a beamformed $M$-element uniform linear array (ULA) as a base station antenna (BSA). The analysis assumes a single sector cell serving two user equipments (UEs) in a downlink multiuser multiple-input multiple-output (MU-MIMO) system employing zeroforcing (ZF) transmission. Determinative design parameters of the BSA configuration are identified. Afterwards, their effects on the power spread across the array are investigated when the positions of the users are random and they are uniformly distributed over the cell. It is shown that the power distribution across the ULA antenna aperture is a periodic function whose characteristics mainly depend on the angular separation of two UEs and the number of BSA antenna elements. A significant variation between ULA input powers is seen to occur if the number of elements in the ULA is smaller than one period of this power distribution function. In order to mitigate the dynamic range of the power variation across the array, an upper bound for the inter-element spacing, depending on the field-of-view (FoV) of the BSA, is defined. It is shown that, in a 20-element ULA, increasing the inter-element spacing from $0.5 \lambda$ to $1.4 \lambda$ reduces the power variation from $19 \mathrm{~dB}$ to $10 \mathrm{~dB}$ for differentiating two close-by UEs with $1^{\circ}$ angular separation.
\end{abstract}

Index Terms-5G, base station antenna, beamforming, massive MIMO, uniform linear array, zero-forcing.

\section{INTRODUCTION}

The continuously increasing demand for higher data rates [1], together with the ambition to connect 50 billion devices by 2020 [2], have stimulated the development of base station antenna (BSA) technology; BSA arrays employing an unprecedentedly large number of active elements operating at mmWave frequencies have been proposed as one of the key $5 \mathrm{G}$ technologies. If using traditional technology, however, the total energy consumption will become a major concern, even more so due to the millions more BSAs that will need to be installed. From the system-level design perspective, energy consumption is a critical metric, which depends on the cellular network layout, the digital modulation, the beamforming algorithm, the (power) electronics and the baseband processing.

The energy consumption in a BSA RF-chain is typically constrained by the relatively poor efficiency of the power amplifiers (PAs). Communication channel models often employ ideal PAs [3], [4]. However, as the integration level increases, the inclusion of more realistic PAs becomes important [5], [6]. Since the PA efficiency depends on its output power, a time-varying and non-uniform amplitude excitation scheme at the BSA ports to control the side lobe levels and/or to realize multi-beam performance will strongly affect the efficiency of the PA. Hereupon, per-antenna power constraints have been recently considered in the optimization algorithms to find an efficient zero-forcing (ZF) precoder [7], [8]. In this sense, it is insightful to first understand how multi-user distribution and antenna

N. Amani, M. V. Ivashina and R. Maaskant are with the Department of Electrical Engineering, Chalmers University of Technology, Gothenburg, Sweden (email: anavid@chalmers.se).

R. Maaskant is also with the Electromagnetics Group, Eindhoven University of Technology (TU/e), The Netherlands.

A. A. Glazunov is with the Department of Electrical Engineering, University of Twente, Enschede, The Netherlands and he is also affiliated with the Department of Electrical Engineering, Chalmers University of Technology, Gothenburg, Sweden. design parameters affect the per-antenna power distribution when conventional $\mathrm{ZF}$ is adopted as a precoder [9].

This paper sheds light on how the multi-user (MU) distribution affects the power allocation of a ZF beamformed uniform linear array (ULA) in a downlink single-cell network. In Sec. II-A a lineof-sight (LOS) environment with perfect channel state information (CSI) is assumed as well as a figure-of-merit of the power variation over the ULA is introduced in Sec. II-B. In Sec. III-A the problem is analytically investigated and a generic closed form expression is derived to calculate the ULA required excitation vector depending on the UE's angular position in the sectorial cell. Also, analytical expressions are presented for the defined figure-of-merits. In Sec. III$\mathrm{B}$ a sparse array with an upperbound on the inter-element spacing is proposed as an alternative to mitigate the spread of power distribution across the array. The performance of the proposed sparse arrays are compared to conventional ULAs by evaluation of a figure-of-merit through simulations. Conclusions are drawn in Sec. IV.

Regarding the notation, boldface upper case and lower case represent matrices and vectors, respectively, while $\mathbb{C}^{M \times K}$ denotes the space of $M \times K$ complex matrices. The Hermitian operator and $L^{2}$ norm are denoted by $(.)^{\dagger}$ and $\|.\|_{2}$, respectively.

\section{System Model}

\section{A. Problem formulation}

We consider the downlink of a single-cell MU multiple-input multiple-output (MIMO) system. The BSA is composed of an $M$ element ULA that communicates with $K$ single-antenna UEs that are randomly located in the BSA's field-of-view (FoV). The received signal vector $\mathbf{y} \in \mathbb{C}^{K \times 1}$ is given by

$$
\mathbf{y}=\mathbf{H W s}+\mathbf{u},
$$

where $\mathbf{s} \in \mathbb{C}^{K \times 1}$ and $\mathbf{H} \in \mathbb{C}^{K \times M}$ are the transmit signal vector to the UEs and the channel matrix, respectively. It is assumed that symbol sequences are uncorrelated with zero mean and unit variance, which results in $\mathbb{E}\left\{\mathbf{s s}^{\dagger}\right\}=I_{\mathrm{K}},[10]$. $\mathbf{W} \in \mathbb{C}^{M \times K}$ is the ZF precoding matrix

$$
\mathbf{W}=\mathbf{H}^{\dagger}\left(\mathbf{H H}^{\dagger}\right)^{-1} .
$$

The UE receiver noise $\mathbf{u} \in \mathbb{C}^{K \times 1}$ satisfies $\mathbf{u} \sim \mathcal{C N}(0, I)$. Assuming LOS propagation, antenna elements with flat-top radiation pattern inside the FoV and zero elsewhere, and perfect CSI, the entries of $\mathbf{H}$, denoted by

$$
h_{k, m} \propto r_{k, m}^{-1} e^{-j \beta r_{k, m}},
$$

can be calculated knowing the Euclidean distance $r_{k, m}=$ $\sqrt{\left(x_{m}-x_{k}\right)^{2}+\left(y_{m}-y_{k}\right)^{2}}$ between the $k$-th UE and the $m$-th BSA element for $m=1, \ldots, M$, and $k=1, \ldots, K$, where $\beta$ is the free-space propagation constant. To have an analytically tractable model, UEs are assumed to be located in the far-field region of the BSA, and the Fraunhofer approximation is applied. Hence, $r_{k, 1} \cong r_{k, 2} \cong \ldots \cong r_{k, m} \cong r_{k}$ for the amplitude dependence and $r_{k, m} \cong r_{k}-(m-1) d \cos \left(\theta_{k}\right)$ for the phase dependence, where $d$ is the BSA inter-element spacing and $\theta_{k}$ is the respective UE's 


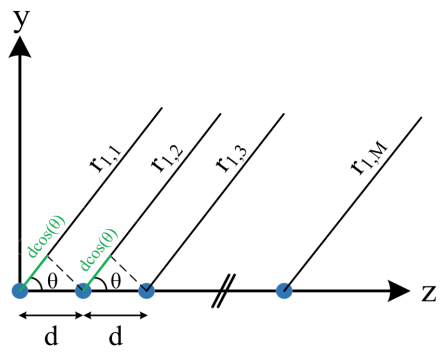

Fig. 1. Fraunhofer approximation in a uniform linear array antenna.

angle toward the ULA, as illustrated in Fig. 1. Under this practical assumption, Eq. (3) is rewritten as

$$
h_{k, m} \propto r_{k}^{-1} e^{-j \beta\left(r_{k}-[m-1] d \cos \theta_{k}\right)} .
$$

The $k$ th column $\mathbf{w}_{k}$ of $\mathbf{W}$ is known as the weight or excitation vector, and corresponds to one beam serving the $k$ th UE. We therefore investigate the effect of the UEs' location and its impact on the required per-antenna power given by $\left|w_{k, m}\right|^{2}$, i.e., the squared absolute value of the elements of vector $\mathbf{w}_{k}$. Increasing $M$ leads to a larger BSA aperture and more degrees of freedom for beamforming, therefore it is a critical design parameter affecting the weight vectors. These design parameters are further scrutinized below.

\section{B. Figures of merit}

We are interested in the per-antenna power distribution (with index $m$ ) across the array for a given user $k$. Considering uncorrelated and unit variance symbol sequences, the required per-antenna power to serve $\mathrm{UE}_{k}$ is defined as follows to ensure equal BSA transmit powers for the analyzed scenarios

$$
P_{k, m}^{\text {norm }}=\left(\frac{\left|w_{k, m}\right|}{\left\|\mathbf{w}_{k}\right\|_{2}}\right)^{2} .
$$

In order to quantify the dynamic range of the power variation, the power ratio figure-of-merit is introduced

$$
P_{\text {ratio }, k} \triangleq \frac{\max \left(\mathbf{p}_{k}^{\text {norm }}\right)}{\min \left(\mathbf{p}_{k}^{\text {norm }}\right)},
$$

where $\mathbf{p}_{k}^{\text {norm }}$ is a vector of the normalized power of the BSA elements and the functions $\max (\mathbf{v})$ and $\min (\mathbf{v})$ take the maximum and minimum of a real-valued vector $\mathbf{v}$, respectively. It is worthwhile to note that the ratio is independent of the normalization in (5).

\section{GENERAL ANALYSIS AND NUMERICAL COMPARISONS}

\section{A. Analytical Investigation}

In this section, we derive the elements of the ZF-precoding matrix $\mathbf{W}$ in (2) and the corresponding figure-of-merit. Considering two UEs in the $M$-element BSA's FoV, and by using (4), the channel matrix $\mathbf{H} \in \mathbb{C}^{2 \times M}$ becomes

$$
\mathbf{H}=\left[\begin{array}{lll}
r_{1}^{-1} e^{-j \beta r_{1}} & \ldots & r_{1}^{-1} e^{-j \beta\left(r_{1}-[M-1] d \cos \theta_{1}\right)} \\
r_{2}^{-1} e^{-j \beta r_{2}} & \ldots & r_{2}^{-1} e^{-j \beta\left(r_{2}-[M-1] d \cos \theta_{2}\right)}
\end{array}\right]
$$

so that $\mathbf{H H}^{\dagger}$ is a Hermitian $2 \times 2$ matrix, with elements that can be straightforwardly shown to be given by

$$
\begin{aligned}
& \left(\mathbf{H H}^{\dagger}\right)_{(1,1)}=r_{1}^{-2} M, \\
& \left(\mathbf{H H}^{\dagger}\right)_{(1,2)}=\left(r_{1} r_{2}\right)^{-1} e^{-j \beta\left[r_{1}-r_{2}\right]} \mathrm{AF}_{12}, \\
& \left(\mathbf{H H}^{\dagger}\right)_{(2,1)}=\left(r_{1} r_{2}\right)^{-1} e^{-j \beta\left[r_{2}-r_{1}\right]} \mathrm{AF}_{21} \\
& \left(\mathbf{H H}^{\dagger}\right)_{(2,2)}=r_{2}^{-2} M
\end{aligned}
$$

where, for further convenience, we have introduced the Array Factor (AF) term

$$
\mathrm{AF}_{12}=\frac{\sin \left(\frac{M}{2} \beta d \psi_{12}\right)}{\sin \left(\frac{1}{2} \beta d \psi_{12}\right)} e^{-j \beta d\left(\frac{M-1}{2}\right) \psi_{12}},
$$

where $\psi_{12}=-\psi_{21}=\cos \theta_{2}-\cos \theta_{1}$ is the path length difference factor due to the angular separation between two users, which can be both positive or non-positive. The array factor satisfies the condition $\mathrm{AF}_{12}=\mathrm{AF}_{21}^{*}$ which is used throughout the derivations. The determinant of $\mathbf{H H}^{\dagger}$ is

$$
\operatorname{det}\left(\mathbf{H H}^{\dagger}\right)=\left(r_{1} r_{2}\right)^{-2}\left(M^{2}-\left|\mathrm{AF}_{12}\right|^{2}\right) .
$$

A non-trivial singularity for $r_{1} \neq \infty$ and $r_{2} \neq \infty$ is seen to occur if

$$
\frac{d}{\lambda} \psi_{12}=n, \quad n=0, \pm 1, \ldots, \pm \infty
$$

Upon assuming $120^{\circ} \mathrm{FoV}$ and $d / \lambda=0.5$ for the BSA, a singularity is seen to be prevented if $\theta_{1} \neq \theta_{2}$. Otherwise, by the properties of the array function, $\mathrm{AF}_{12}, \mathrm{AF}_{21} \rightarrow M$ as $\psi_{12}, \psi_{21} \rightarrow 0$ and a singularity is seen to occur.

On account of (2), the weight vector entries $w_{1, m}$ corresponding to the first UE, for $m=1,2, \ldots, M$, can now be calculated analytically as

$$
w_{k, m}=\frac{r_{k} M e^{j \beta\left(r_{k}-[m-1] d \cos \theta_{k}\right)}}{M^{2}-\left|\mathrm{AF}_{k l}\right|^{2}}\left(1-\frac{\mathrm{AF}_{l k}}{M} e^{-j[m-1] \beta d \psi_{k l}}\right),
$$

where $k \neq l \in\{1,2\}$.

Inserting (12) into (5) we obtain the following result for the power variation across the array in the dual-UE case

$$
P_{k, m}^{\mathrm{norm}}=\frac{\left|1-\frac{\mathrm{AF}_{l k}}{M} e^{-j[m-1] \beta d \psi_{k l}}\right|^{2}}{M\left(1-\frac{\left|\mathrm{AF}_{k l}\right|^{2}}{M^{2}}\right)} .
$$

An upper bound of the corresponding power ratio figure-of-merit can then be obtained in a compact form as

$$
P_{\text {ratio }, k}=\left(\frac{M+\left|\mathrm{AF}_{k l}\right|}{M-\left|\mathrm{AF}_{k l}\right|}\right)^{2},
$$

where $k \neq l \in\{1,2\}$. In obtaining (14) we applied the triangle inequality to the numerator of (13) to obtain the maximum, i.e., $\mid z_{1}+$ $z_{2}|\leq| z_{1}|+| z_{2} \mid$ and the reverse triangle inequality to obtain the minimum, i.e., $\left|z_{1}-z_{2}\right| \geq|| z_{1}|-| z_{2}||$. Hence, while (12) and (13) are exact under the Fraunhofer approximation, (14) is not exact in general. Employing the triangle inequalities, the numerator of (14) is overestimated while the denominator is underestimated. This results in a higher value for the upper bound than the actual exact value.

Let us first consider the important asymptotic case for extremely large $M$, for which we have that

$$
w_{k, m} \approx \frac{r_{k}}{M} e^{j \beta\left(r_{k}-[m-1] d \cos \theta_{k}\right)}=\frac{1}{M h_{k, m}} .
$$

Hence, in the limiting case, the weights are the inverses of the corresponding channel matrix entries. The phase depends upon the element index $m$ and is the conjugate of the phase of the corresponding signal, while the amplitudes equalize. Therefore, for extremely large $M$, we have that $P_{1, m}^{\text {norm }}=P_{2, m}^{\text {norm }}=\frac{1}{M}$ and also $P_{\text {ratio }}=1$. The same result can be obtained from (13) and (14). Hence, we can conclude that, Massive-MIMO (large $M$ ) will asymptotically be favorable with respect to the PA output power characteristics.

On the other hand, practical antenna systems employ a finite number of antenna elements. Adjusting side lobe level and/or creating multiple simultaneous beams therefore incurs a power variation across the elements of the BSA array. Based on (13), the variation of 


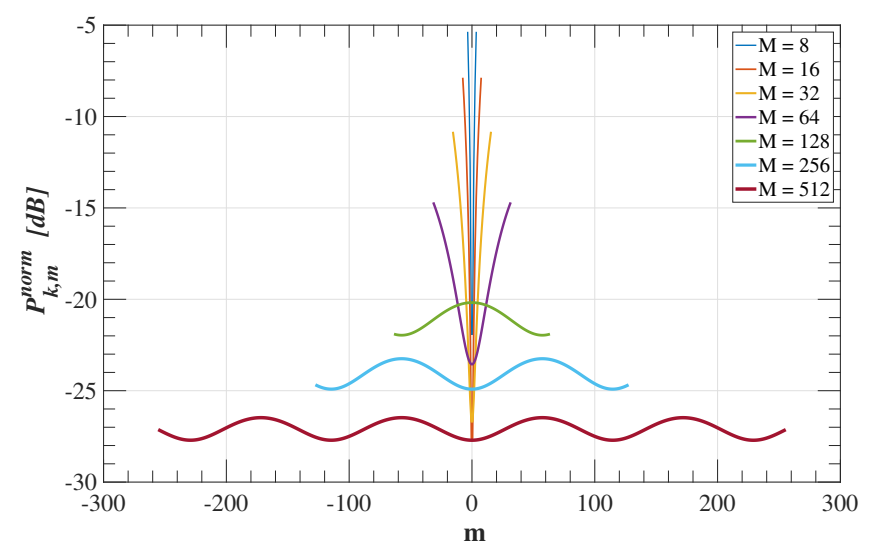

Fig. 2. Normalized power distribution at the ULA antenna comprising different number of elements $M$ for $\left|\psi_{12}\right|=0.0175, \theta_{1}=89.5^{\circ}$, and $\theta_{2}=90.5^{\circ}$. Results are shown for $d / \lambda=0.5$.

the power across the array as a function of the index $m$ is given solely by the complex exponent expression. In this case, the fundamental period of the phase variation in (3), taking $m$ as an integer into account, is

$$
T_{m}=\left\lfloor\frac{2 \pi}{\beta d\left|\psi_{12}\right|}\right\rfloor,
$$

where $\lfloor x\rfloor$ denotes the floor function, i.e., the integer part of $x \in \mathbb{R}$. Hence, the coefficient will have a non-periodic behavior if $M<T_{m}$,

$$
M<\left\lfloor\frac{\lambda}{d\left|\psi_{12}\right|}\right\rfloor \text {. }
$$

Otherwise, the power distribution will vary periodically with the index $m$, or more precisely, the variation will be a truncated sinusoidal function with period $T_{m}$. The power ratio will decrease as $M$ increases, which follows from (14), but this is traded against having more active elements in the array.

Fig. 2 shows the normalized per-antenna power distribution across the ULA antenna, Eq. (13), with $d / \lambda=0.5$, for different number of elements $M$. Two UEs are assumed to be located close-by where $\theta_{1}=89.5^{\circ}$ and $\theta_{2}=90.5^{\circ}$, hence $\left|\psi_{12}\right| \approx 0.0175$. This very small angular separation causes highly correlated channel vectors or equivalently an ill-conditioned $\mathbf{H H}^{\dagger}$. In this case a significant power variation occurs when the antenna array becomes deficient in terms of the number of elements. Therefore, a huge number $\mathrm{M}$ is needed to lower the power variation across the antennas, while preserving the distinguishment between the UEs. Results show a clear dependence of the power across the array that changes in shape depending on the number of antenna elements $M$. Although the non-periodic behaviour persists for all $M<T_{m}$, for $M>T_{m}$ a truncated sinusoidal power distribution is achieved.

Fig. 3 shows the power ratio figure-of-merit as a function of the number of elements $M$ for two extreme cases, i.e., for very small and large angular separations, when $d / \lambda=0.5$ and the FoV is $120^{\circ}$. As expected, a very large number of antenna elements is needed for small angular separations, or a significant power variation is required otherwise. Fig. 3(b) shows similar results, but in this case it is assumed that we have the maximum possible separation of $120^{\circ}$, i.e., $\theta_{1}=30^{\circ}$ and $\theta_{2}=150^{\circ}$. As can be seen, the PA power variation is much smaller since the $\mathrm{ZF}$-algorithm can more easily resolve the angles of the two UEs. Therefore, a situation where two users are located close-by constitutes the worst case. In addition, the upper bound in (14) becomes tight at lower values of $M$.

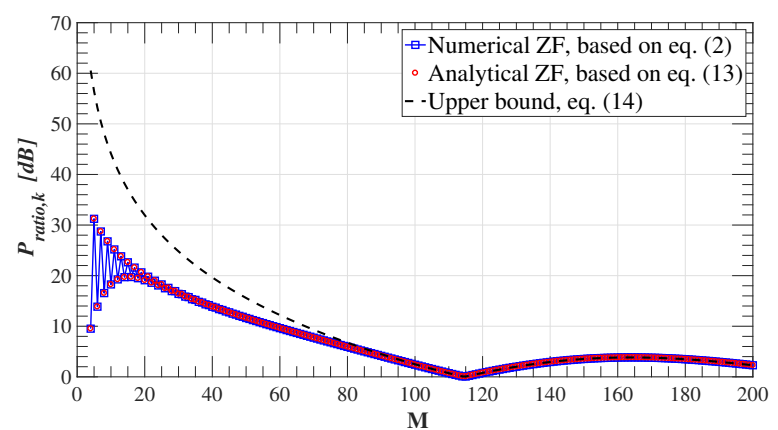

(a)

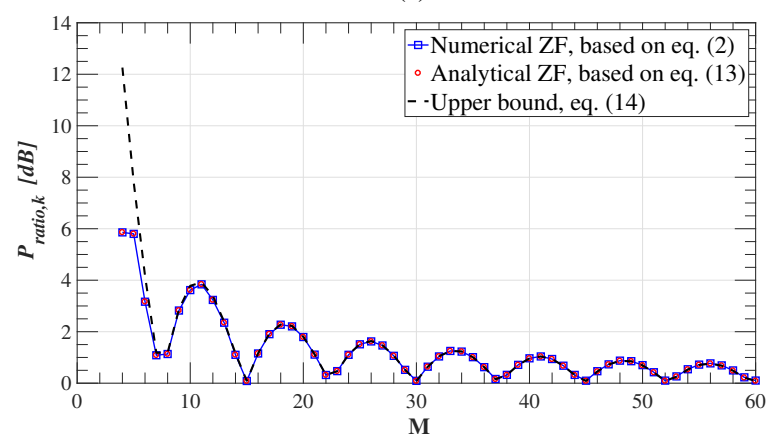

(b)

Fig. 3. Power ratio figure-of-merit calculated analytically and numerically as a function of the number of elements $M$ for different cases, (a) $\theta_{1}=89.5^{\circ}$ and $\theta_{2}=90.5^{\circ}$, (b) $\theta_{1}=30^{\circ}$ and $\theta_{2}=150^{\circ}$. Results are shown for $d / \lambda=0.5$.

\section{B. Sparse Arrays and Performance Comparison}

Spatial distinguishment of UEs is favourable in a $5 \mathrm{G}$ MU-MIMO system by means of multiple BSA beams. As it is shown in Fig. 3(a), a very large number of antenna elements at the linear BSA is required to differentiate close-by UEs for small $P_{\text {ratio }}$. However, increasing $M$ in order to mitigate the variation of the input power distribution at the BSA is a costly solution due to the increased number of active components. It also exacerbates the complexity of the BSA design from a system-level point of view. On account of (16), another parameter which can potentially reduce the power variation at the BSA is the inter-element spacing $d$ in the array. A larger $d$ reduces the periodicity in (16) and therefore a smaller variation in PA powers can be achieved for a given $M$, e.g. by employing sparse arrays. However, Eq. (11) suggests that other singularity conditions can occur by increasing $d$.

In (11), $n= \pm 1$ satisfies the second singularity condition which is undesirable since the ZF-precoder becomes totally incapable to differentiate between UEs. In order to prevent this, a maximum allowable inter-element spacing $\left(d_{\text {sparse }}\right)$ can be defined by the maximum possible angular separation between two UEs in the FoV of the BSA. Since there is not any control on the location of the UEs, increasing $d$ to a value larger than $d_{\text {sparse }}$ might lead to a singularity for certain angular separations that are not necessarily small, which equivalently results in a significant power variation. Therefore, an upper bound for $d_{\text {sparse }}$ can be defined as

$$
0.5 \lambda<d_{\text {sparse }}<\frac{\lambda}{\left|\psi_{\max }\right|},
$$

where $\left|\psi_{\max }\right|$ directly depends on the FoV of the BSA. Actually, (18) guarantees that no grating lobes exist in the FoV of the sparse BSA, hence the lower bound. 


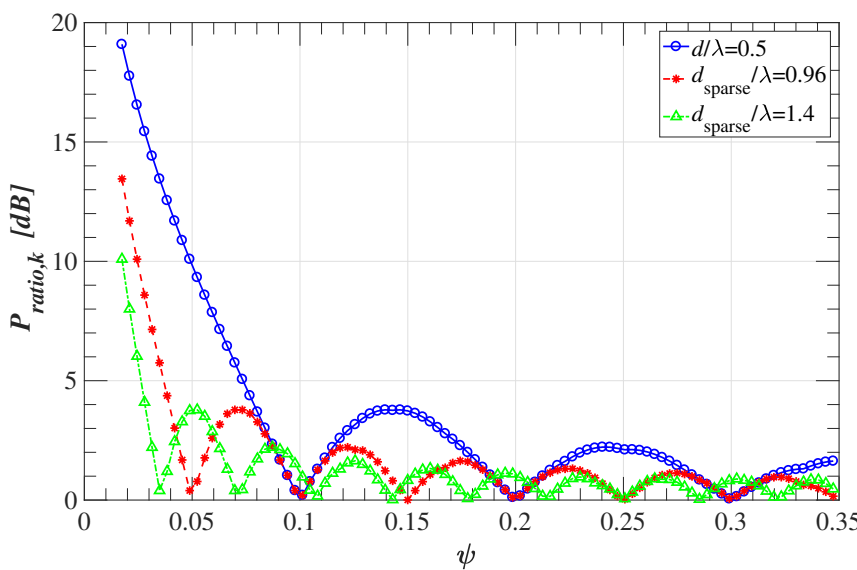

Fig. 4. Power ratio figure-of-merit for different inter-element spacings $(d)$ in a 20-element linear BSA as a function of angular separation.

Traditionally, a BSA is a sectorized antenna with three panels each covering $120^{\circ}$. In this way $\left|\psi_{\max }\right|=1.73$ and therefore $\left.d\right|_{\mathrm{FoV}=120^{\circ}}<0.578 \lambda$. Hence, there is not much of a possibility to increase the inter-element spacing for such a large FoV. However, multiple panels with smaller FoVs can be utilized to cover one sector of the BSA, e.g. two or three panels each having $60^{\circ}$ or $40^{\circ} \mathrm{FoV}$, respectively. Then, $\left|\psi_{\max }\right|$ reduces to 1 and 0.68 for these reduced FoVs and therefore $\left.d_{\text {sparse }}\right|_{\mathrm{FoV}=60^{\circ}}<\lambda$ and $\left.d_{\text {sparse }}\right|_{\mathrm{FoV}=40^{\circ}}<1.47 \lambda$.

In order to evaluate the $P_{\text {ratio }}$ figure-of-merit in a sparse array realized by (18), a worst-case scenario with two UEs are considered to be located very close to each other $\left(\theta_{1}=89.5^{\circ}\right.$ and $\left.\theta_{2}=90.5^{\circ}\right)$, in a same distance from a 20-element BSA. Afterwards, they are symmetrically moved away from each other to increase the angular separation while their distance to the BSA remains the same. Results are extracted for $\left.d\right|_{\mathrm{FoV}=120^{\circ}}=0.5 \lambda,\left.d_{\text {sparse }}\right|_{\mathrm{FoV}=60^{\circ}}=0.96 \lambda$ and $\left.d_{\text {sparse }}\right|_{\mathrm{FoV}=40^{\circ}}=1.4 \lambda$, see Fig. 4 , for different $\psi$ values. It is concluded that, for small $\psi$ values, where two UEs are located very close to each other and where a regular array is becoming deficient due to the insufficient number of antenna elements, a sparse array is a sensible alternative to reduce the $P_{\text {ratio. For instance for a } 1^{\circ}}$ angular separation, the corresponding $P_{\text {ratio }}$ of $19 \mathrm{~dB}$ achieved by a regular array is reduced to $13.4 \mathrm{~dB}$ and $10 \mathrm{~dB}$ by sparse arrays with $60^{\circ}$ and $40^{\circ} \mathrm{FoV}$, respectively.

The $P_{\text {ratio }}$ figure-of-merit is also illustrated in Fig. 5 as contour plots for $\left.d\right|_{\mathrm{FoV}=120^{\circ}}=0.5 \lambda$ and $\left.d_{\text {sparse }}\right|_{\mathrm{FoV}=40^{\circ}}=1.4 \lambda$, for different number of antenna elements and $\psi$ values. As can be seen, increasing the inter-element spacing can mitigate the $P_{\text {ratio }}$ for all $M$ when $\psi$ is small, but the improvement is more pronounced for smaller $M$ values. This is due to the larger antenna aperture and spatial resolution enhancement of the sparse array which is shown to outperform the regular array for the case of closely separated users.

\section{CONCLUSION}

An analytical model has been presented to calculate the per-antenna power spread for an $M$-element ULA serving two UEs employing the ZF-precoder. The power distribution across the ULA turns out to be a periodic function whose characteristics mainly depend on the angular separation between two UEs and the number of antenna elements in the BSA. A significant power variation has been observed at the BSA ports in order to differentiate two close-by UEs. Increasing the number of BSA elements has shown to reduce the power variation along the array, however, it adds cost and complexity to the system. It has furthermore been shown that the periodicity of the power distribution function can also be adjusted by the inter-element spacing
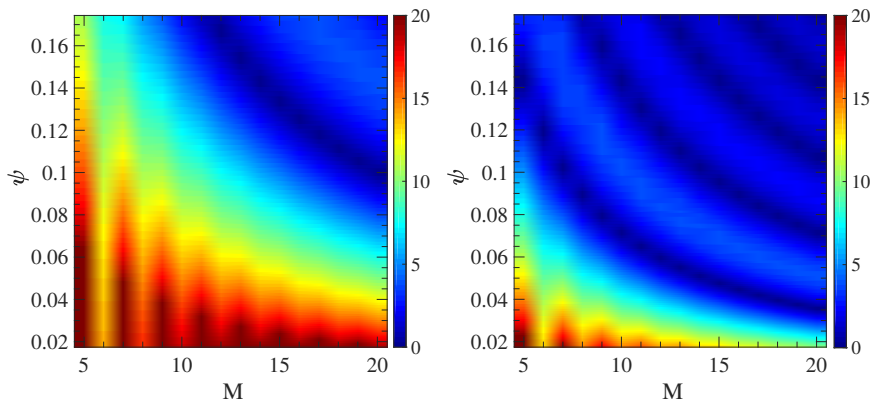

Fig. 5. Power ratio figure-of-merit as a function of the number of elements $M$ and angular separation $\psi$ for different inter-element spacings, (left) $d=$ $0.5 \lambda$, (right) $d_{\text {sparse }}=1.4 \lambda$.

in the BSA to reduce the per antenna power variation; sparse arrays with the inter-element spacings larger than $0.5 \lambda$ have been suggested for this purpose. In order to prevent $\mathrm{ZF}$ singularity problems due to grating lobes, an upper bound for the inter-element spacing, depending on the corresponding FoV, has been defined. It has been illustrated that, the dynamic range of the power variation across a 20-element BSA can be reduced from $19 \mathrm{~dB}$ to $10 \mathrm{~dB}$ with the aid of a sparse array having $d=1.4 \lambda$ and $\mathrm{FoV}=40^{\circ}$ to distinguish two UEs with $1^{\circ}$ angular separation. It is therefore concluded that a sparse array, due to its spatial resolution enhancement, is capable of outperforming a regular array in terms of distinguishing between close-by UEs.

\section{ACKNOWLEDGMENT}

This project has received funding from the European Union's Horizon 2020 research and innovation programme under the Marie Sklodowska-Curie grant agreement No 721732.

\section{REFERENCES}

[1] Qualcomm, "The 1000x mobile data challenge," Available: https://www.qualcomm.com/documents/1000x-mobile-data-challenge, 2013.

[2] Ericsson, "More than 50 billion connected devices," White Paper, 2011.

[3] J. Xu and L. Qiu, "Energy efficiency optimization for MIMO broadcast channels," IEEE Transactions on Wireless Communications, vol. 12, no. 2, pp. 690-701, 2013.

[4] D. Nguyen, L.-N. Tran, P. Pirinen, and M. Latva-aho, "Precoding for full duplex multiuser MIMO systems: Spectral and energy efficiency maximization," IEEE Transactions on Signal Processing, vol. 61, no. 16, pp. 4038-4050, 2013.

[5] Y. Dong, Y. Huang, and L. Qiu, "Energy-efficient sparse beamforming for multiuser MIMO systems with nonideal power amplifiers," IEEE Transactions on Vehicular Technology, vol. 66, no. 1, pp. 134-145, 2017.

[6] D. Persson, T. Eriksson, and E. G. Larsson, "Amplifier-aware multipleinput single-output capacity," IEEE Transactions on Communications, vol. 62, no. 3, pp. 913-919, 2014.

[7] J. Jang, S.-W. Jeon, H. Chae, H.-S. Cha, and D. K. Kim, "Low complexity zeroforcing precoder design under per-antenna power constraints," IEEE Communications Letters, vol. 19, no. 9, pp. 1556-1559, 2015.

[8] T. M. Pham, R. J. Farrell, J. Dooley, E. Dutkiewicz, D. N. Nguyen, and L.-N. Tran, "Efficient zero-forcing precoder design for weighted sumrate maximization with per-antenna power constraint," IEEE Transactions on Vehicular Technology, 2017.

[9] C. Bencivenni, A. A. Glazunov, R. Maaskant, and M. V. Ivashina, "Effects of regular and aperiodic array layout in multi-user mimo applications," in Antennas and Propagation \& USNC/URSI National Radio Science Meeting, 2017 IEEE International Symposium on. IEEE, 2017, pp. 1877-1878.

[10] H. Yang and T. L. Marzetta, "Performance of conjugate and zero-forcing beamforming in large-scale antenna systems," IEEE Journal on Selected Areas in Communications, vol. 31, no. 2, pp. 172-179, 2013. 